\title{
Prótese obturadora palatina: opção reabilitadora para paciente oncológico maxilectomizado
}

\author{
Palatal obturator: rehabilitator option for oncological maxilectomized patient \\ Prótesis obturadora palatina: opción rehabilitadora para paciente oncológico maxilectomizado
}

\author{
Gabriela Manucci DE MELLO' \\ Marcella Tassi FARINA ${ }^{1}$ \\ Sabrina Paiva Guedes HENRIQUE ${ }^{1}$ \\ Marcela Filié HADDAD ${ }^{2}$
}

${ }^{1}$ Cirurgiã-Dentista, Departamento de Odontologia Restauradora, Faculdade de Odontologia da Universidade Federal de Alfenas (UNIFAL)37.130-000, Alfenas-MG, Brasil

${ }^{2}$ Cirurgiã-Dentista, Mestre e Doutora em Odontologia - Área de Concentração: Prótese Dentária. Professora de Prótese Total Removível,

Departamento de Odontologia Restauradora, Faculdade de Odontologia da Universidade Federal de Alfenas (UNIFAL) 37.130-000, Alfenas - MG, Brasil

\section{Resumo}

Introdução: O tratamento de câncer de boca muitas vezes mutila o paciente fazendo com que o mesmo perca dentes, parte de estrutura de palato, língua ou assoalho, dificultando sua mastigação e fonação, resultando em problemas nutricionais e excluindo-o do convívio social. Neste sentido, a reabilitação protética desse paciente torna-se cada vez mais relevante e tem por objetivo restaurar a estética, função e autoestima perdidas. Objetivo: relatar um caso clínico a respeito da atuação do cirurgião dentista na reabilitação protética de paciente que sofreu maxilectomia parcial decorrente de remoção de tumor. Descrição do caso clinico: Foi confeccionada uma prótese parcial removível obturadora palatina para o arco maxilar e uma prótese parcial removível convencional para o arco mandibular. Conclusão: Este tipo de reabilitação promove restauração oclusal, estética e fonética ao paciente, resultando em melhora da qualidade de vida e permitindo seu retorno ao convívio social sem constrangimentos.

Descritores: Neoplasias Bucais; Prótese Maxilofacial; Anormalidades Maxilofaciais; Obturadores Palatinos.

\section{Abstract}

Introduction: The treatment of mouth cancer often mutilates the patient causing him to lose teeth, part of the structure of the palate, tongue or floor, making it difficult to chew and phonate, resulting in nutritional problems and excluding social interaction. In this sense, the prosthetic rehabilitation of this patient becomes increasingly relevant and aims to restore lost aesthetics, function and self-esteem. Objective: to report a clinical case regarding the performance of the dental surgeon in the prosthetic rehabilitation of a patient who underwent partial maxilllectomy due to tumor removal. Clinical Report: Partial Removable Palatal Obturator Prosthesis was made for the maxillary arch and a conventional removable partial denture for the mandibular arch. Conclusion: This type of rehabilitation promotes occlusal, aesthetic and phonetic restoration to the patient, resulting in improvement of the quality of life and allowing its return to the social life without constraints. Descriptors: Mouth Neoplasms; Maxillofacial Prosthesis; Maxillofacial Abnormalities; Palatal Obturators.

\section{Resumen}

Introducción: El tratamiento de cáncer de boca a menudo mutila al paciente haciendo que el mismo pierda dientes, parte de estructura de paladar, lengua o piso, dificultando su masticación y fonación, resultando en problemas nutricionales y excluyendo de la convivencia social. En este sentido, la rehabilitación protética de ese paciente se vuelve cada vez más relevante y tiene por objetivo restaurar la estética, función y autoestima perdidas. Objetivo: relatar un caso clínico acerca de la actuación del cirujano dentista en la rehabilitación protética de paciente que sufrió maxilectomía parcial resultante de remoción de tumor. Descripción del caso clínico: Se ha confeccionado una prótesis parcial removible obturadora palatina para el arco maxilar y una prótesis parcial removible convencional para el arco mandibular. Conclusión: Este tipo de rehabilitación promueve restauración oclusal, estética y fonética al paciente, resultando en mejora de la calidad de vida y permitiendo su retorno a la convivencia social sin limitaciones.

Descriptores: Neoplasias de la Boca; Prótesis Maxilofacial; Anomalias Maxilofaciales; Obturadores Palatinos.

\section{INTRODUÇÃO}

O palato é a estrutura responsável por separar a cavidade nasal da oral. A perda de estrutura do palato pode estar associada a problemas congênitos, traumatismos ou tumores. A fissura palatal é uma deformidade associada ao lábio leporino, causada por uma anomalia congênita que ocorre devido à má formação fetal e que causa graves problemas na estrutura oronasal ${ }^{1}$. A perda por trauma ocorre devido a acidentes e pode gerar problemas nos sistemas digestivo e respiratório do paciente, acometendo fala, deglutição e respiração ${ }^{2}$. Já a perda por tumor, ocorre devido à cirurgia de retirada do mesmo, e considerando-se a sua extensão, podem ser muito mutiladoras, com sequelas ao paciente e a sua qualidade de vida ${ }^{3}$.

O carcinoma epidermóide é o tumor que mais acomete a região intraoral $\mathrm{e}$, devido à dificuldade para o estabelecimento de seu diagnóstico, quando descoberto, estruturas adjacentes a ele já foram acometidas e acabam sendo perdidas nas maxilectomias, gerando graves consequências ao paciente ${ }^{4}$.
Estas consequências têm grande impacto não somente na aparência do paciente, mas também sobre o aspecto psicossocial do mesmo ${ }^{5}$. A maxilectomia, por ser mutiladora, faz com que se perca a capacidade de mastigação e fonação normal, sendo que esta fica hipernasalada. A alimentação também é prejudicada, pois há regurgitação de alimentos e líquidos da cavidade oral para a cavidade nasal, o que gera transtornos na vida do paciente ${ }^{6}$. Isso faz com que surjam problemas psicológicos como depressão, o que acaba prejudicando sua interação social ${ }^{7}$.

Fica clara então, a necessidade da atuação de uma equipe multidisciplinar capaz de ajudar o paciente durante suas necessidades em todas as áreas, atuando nele como um todo ${ }^{8}$. O defeito cirúrgico criado no tratamento de lesões traumáticas ou neoplásicas pode levar, além das consequências citadas acima, também, a deformidade estética severa, sendo fundamental a sua correção cirúrgica ou protética 9 .

Em tratamentos protéticos, são utilizadas próteses bucomaxilofaciais, que podem ser intraorais, 
extraorais ou combinadas. As intraorais compreendem as próteses obturadoras palatinas, bucofaringeanas, mandibulares e de língua. As extraorais são as faciais, sendo oculares, nasais, auriculares e oculopalpebrais. Já as combinadas associam dispositivos de reabilitação intra e extraorais ${ }^{10}$. As próteses obturadoras têm por objetivo ocluir os defeitos maxilares e restaurar as regiões orofaríngeas, ósseas orbitais e até mesmo da base do crânio, restabelecendo as funções mastigatórias, fonação e estética do paciente ${ }^{11,12}$.

Assim, o presente estudo tem como objetivo relatar um caso clínico a respeito da atuação do cirurgião dentista na reabilitação protética de paciente que sofreu maxilectomia parcial decorrente de remoção de tumor.

\section{CASO CLÍNICO}

Inicialmente, o projeto foi encaminhado ao Comitê de Ética em Pesquisa da Universidade Federal de Alfenas (CEP/ UNIFAL-MG) e obteve parecer favorável (Parecer ${ }^{\circ}$ 2.544.252).

$\mathrm{O}$ caso clínico relatado se trata da reabilitação protética de um paciente que passou por tratamento oncológico. O paciente J.D.S.S, sexo masculino, leucoderma, 48 anos, procurou atendimento na clínica de Prótese Total Removível da UNIFAL-MG para a confecção de uma prótese obturadora palatina.

Durante a anamnese, o paciente relatou trabalhar como gesseiro, e não possuir nenhuma doença crônica. Ele mencionou ter feito uso de cigarro durante 33 anos da sua vida, mas após a cirurgia para retirada da maxila parou de fumar. Afirmou não ingerir bebida alcoólica e nem drogas de abuso, e os medicamentos que utiliza são para controle do câncer.

Relatou, ainda, ter procurado a Clínica Integrada da Universidade Federal de Alfenas em julho de 2016, devido a uma dor no dente 27. O elemento foi então extraído e nos dias seguintes apresentou-se uma secreção purulenta e dificuldade de cicatrização no local. Foi constatada também a presença de comunicação bucosinusal. Ele então procurou um cirurgião-dentista um tempo depois, sendo que o paciente não soube relatar o tempo ao certo, que observou uma tumefação na região do dente extraído com aparência sugestiva de tumor e, então, encaminhou o paciente a um estomatologista.

O paciente foi então submetido a uma biópsia da região afetada, em que constatou-se tratar de um tumor maligno. $\mathrm{O}$ exame histopatológico fez a descrição microscópica e macroscópica do tumor, no qual se obteve o laudo de um carcinoma espinocelular moderadamente diferenciado, da região maxilar, sendo os planos cirúrgicos coincidentes com a lesão. Dessa forma, o paciente foi imediatamente encaminhado para o Cirurgião de Cabeça e Pescoço, profissional adequado para realizar o tratamento.
Devido à demora nos exames, o paciente relatou que houve o crescimento do tumor, o que acarretou a necessidade de uma cirurgia mais invasiva para realizar a sua remoção, causando assim a perda da maxila e de parte do palato. Feita a cirurgia, foram realizados novos exames, entre eles de ressonância magnética que concluiu haver o controle de status pós-operatório de ressecção de tumor maxilar à esquerda, com alterações acima pormenorizadas (dado clínico). E presença de sinusopatia frontoetmoidal à esquerda e maxilar à direita.

Depois da cirurgia o paciente relatou que teve dificuldades na fala, que ficou de difícil compreensão e também na mastigação, o que ocasionou a perda de $23 \mathrm{~kg}$. Após o tratamento cirúrgico, o paciente retornou à UNIFAL para realizar a avaliação da sua cavidade bucal e o estado dos seus dentes, procedimento comum em pacientes que serão submetidos à radioterapia. Foi então realizado o tratamento, efetuando-se as extrações necessárias antes do início da radioterapia.

Em seguida, o paciente passou por 33 sessões de radioterapia e 9 sessões de quimioterapia. Após o tratamento, o paciente recebeu alta, tendo sintomas leves de dor local, não apresentando mais evidência da doença. Assim, foi encaminhado para realizar a confecção de uma prótese obturadora palatina, importante etapa na sua reabilitação e no seu tratamento.

Durante o exame clínico extraoral foi notada assimetria facial; perda da continuidade da pele do lado esquerdo da face e redução da abertura bucal decorrente de fibrose cicatricial. No exame clínico intraoral foi observada extensa perda de estrutura do palato do lado esquerdo da maxila (Figura 1); ausência dos elementos 21, 22, 23, 24, 25, 26 e 27, retirados em decorrência da maxilectomia parcial, e dos elementos 11, 15, 16, 34, 35 e 44. Os elementos 12,13 e 14 encontravam-se presentes e eram pilares de um obturador palatino provisório. Foi notado desgaste por bruxismo nos dentes anteriores inferiores e lesão periapical no elemento 45 .

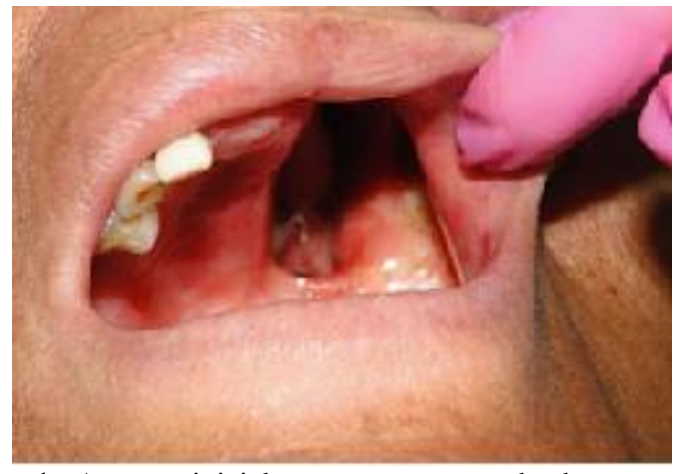

Figura 1: Aspecto inicial, com extensa perda de estrutura do palato do lado esquerdo.

Dessa forma, inicialmente, foi realizado o tratamento endodôntico dos elementos comprometidos e optou-se pela confecção de uma 
prótese parcial removível obturadora palatina para o arco maxilar e uma prótese parcial removível convencional para o arco mandibular seguindo o protocolo estabelecido por Rezende et al. ${ }^{10}$.

Inicialmente, foi realizada a moldagem de estudo. Para a moldagem da maxila (Figura 2), a área de perda de estrutura do palato foi protegida com uma gaze embebida em soro fisiológico (Figura $2 \mathrm{~A}$ ) para evitar a entrada de excesso de material de moldagem no defeito. Em seguida, foi selecionado o par de moldeiras de estoque. As mesmas tiveram suas bordas individualizadas com cera periférica. $\mathrm{O}$ material de escolha para moldagem de estudo foi o alginato (Dentsply Indústria e Comércio ltda, Petrópolis, RJ) que foi proporcionado e manipulado seguindo as instruções do fabricante, inserido nas moldeiras, que foram levadas à boca, sendo então aguardada a geleificação (Figura 2B) do alginato para a remoção dos moldes, checagem da qualidade da impressão tomada (Figura 2C) e obtenção dos modelos de estudo.

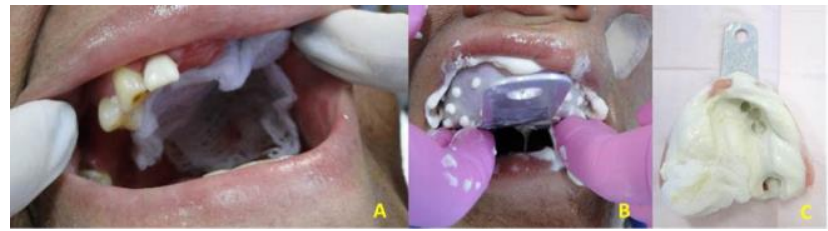

Figura 2: Moldagem de estudo da maxila. A: posicionamento da gaze; B: moldagem propriamente dita; C: molde de estudo.

Após a obtenção do modelo de estudo, foi solicitada a confecção de moldeiras individuais devido à dificuldade de inserção das moldeiras de estoque na boca do paciente. Estes modelos também foram utilizados para o delineamento e planejamento do caso em questão.

Em seguida, foram realizados os preparos específicos, que consistiram em nichos preparados na face oclusal dos elementos 14 (mesial), 17 (distal), 37 (mesial) e 45 (mesial) e nos cíngulos dos dentes 12, 13,33 e 43.

A etapa seguinte consistiu na moldagem funcional dos arcos. Esta foi realizada da mesma maneira que a moldagem de estudo, porém, utilizando as moldeiras individuais. Sobre os modelos de trabalho obtidos a partir desta moldagem foram desenhadas as armações metálicas para fundição.

Em posse das armações fundidas, foi realizada a prova clínica das mesmas, tomados os registros para montagem dos modelos em articulador semiajustável e os dentes artificiais foram selecionados.

O passo seguinte consistiu na prova estética e funcional, onde as próteses com os dentes montados em cera sobre uma base de resina acrílica foram provadas no paciente e então analisada sua estética (confirmando se a cor, formato e tamanho dos dentes estavam satisfatórios e se a oclusão estava correta) e também a função (observando se a pronúncia do paciente estava adequada). Durante o teste fonético foi avaliada a necessidade de aumento do bulbo da prótese obturadora a fim de permitir menor passagem de ar durante a fala. Desse modo, foi realizada a moldagem corretiva (Figura 3) utilizando a técnica da boca fechada, a base da prótese como moldeira e silicone de condensação de consistência leve (Oranwash L, Zhermack, Badia Polesine, Rovigo, Itália).

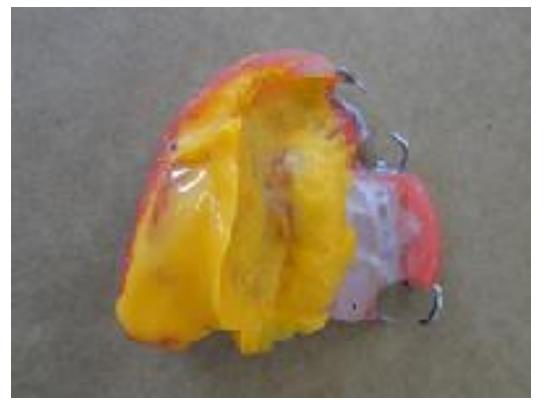

Figura 3: Resultado da moldagem corretiva utilizando a base de prova como moldeira.

Feitas todas as adaptações necessárias, foi selecionada a cor da base acrílica utilizando-se a escala de cores de gengiva (STG - VIPI, Pirassununga, São Paulo, Brasil) e as próteses foram enviadas ao laboratório para acrilização. As próteses finalizadas foram instaladas no paciente (Figura 4). Foi analisada a estabilidade e a retenção das duas próteses (Figura 4-A), Os testes fonéticos foram repetidos e notou-se a pronúncia ainda dificultada. Então, observou-se que a porção posterior do defeito palatino não havia sido vedada. Dessa maneira, procedeu-se ao reembasamento da base da prótese maxilar com reembasador hard (Kooliner, GC America Inc, Alsip, Illinóis, EUA). O reembasamento promoveu completa vedação do defeito maxilar (Figura 4-B).

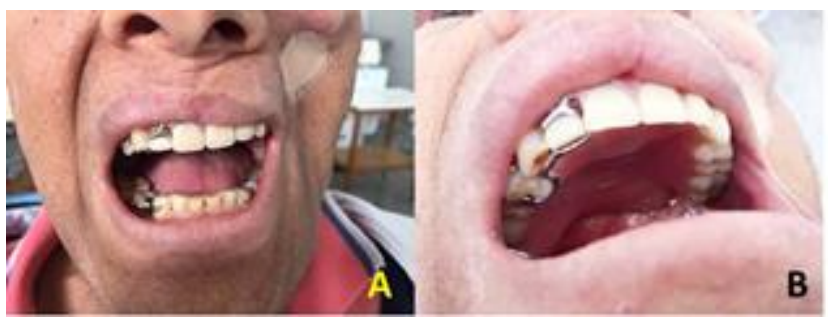

Figura 4: Aspecto final após a instalação das próteses. A: Prótese obturadora palatina maxilar e Prótese Parcial Removível mandibular em posição. B: Defeito palatino completamente vedado.

Em seguida, as bases foram ajustadas com auxílio de pasta evidenciadora de contato e maxicut. Também foram checados os contatos oclusais em fechamento, lateralidade e protrusão mandibular com auxílio de fita de carbono e os desgastes necessários foram realizados.

O paciente recebeu orientações a respeito da forma de uso da prótese, de como colocá-la e retirá-la e que ela não deve ser usada à noite, para permitir que a gengiva e os dentes que a suportam descansem. 
Nesse período deve ser colocada dentro de um copo com água. E sobre a higienização, foi orientado a limpar as próteses após todas as refeições, incluindo a parte interna e os grampos, assim como os dentes remanescentes, e a escovar a prótese com uma escova macia utilizando sabão neutro ou sabonete, evitando o uso de creme dental. Foi ressaltado que as próteses não devem ser fervidas para não danificar a parte de acrílico e que não se deve deixá-las de molho em produtos de limpeza, como por exemplo, água sanitária, que pode provocar danos à estrutura de metal da mesma. Além disso, foi instruído da importância dos retornos periódicos. O paciente foi também aconselhado para que fizesse fisioterapia para recuperar a abertura normal de boca e, dessa maneira, possibilitar o aumento do bulbo obturador com a intenção de melhorar a emissão dos fonemas anasalados.

\section{DISCUSSÃO}

O carcinoma epidermóide em seio maxilar afeta especialmente adultos, na faixa etária entre 50 e 60 anos. Tem predisposição pelo sexo masculino, e é frequentemente assintomático, podendo ser confundido com algumas outras condições, como dor de dente e sinusite ${ }^{13}$. O caso clínico apresentado corrobora com a literatura, sendo que o paciente tem 48 anos de idade, é do gênero masculino e seu sintoma inicial foi uma dor no dente 27 , de forma que somente após sua extração, má cicatrização, presença de dor e secreção purulenta o levaram a procurar novo atendimento, e após novos exames foi descoberto o carcinoma espinocelular maxilar.

É importante ressaltar também que o paciente é gesseiro profissão que apresenta fator de risco para esse tipo de câncer, pois o pó do gesso é considerado um carcinógeno ambiental, como demonstrado na literatura, aumentando $\mathrm{o}$ fator de risco para $\mathrm{o}$ paciente ${ }^{4}$.

Outro ponto relevante é o uso do cigarro, que é considerado um dos fatores de risco para o CEC segundo a literatura ${ }^{4}$, sendo que o paciente é exfumante, e utilizou o tabaco por 33 anos, ratificando mais uma vez os achados literários, e aumentando a sua predisposição a doença.

Além disso, a falta de sintomas, a permanência sem diagnóstico por algum tempo, o uso de medicamentos para dor, que mascararam o problema ${ }^{4}$ foram outros fatores que vão de encontro a casos relatados na literatura. Como esse tumor em maxila é de difícil diagnóstico, quando descoberto normalmente apresenta uma grande extensão, acometendo estruturas adjacentes, como o palato por exemplo, acarretando a sua perda total ou parcial ${ }^{4}$. No caso em questão houve perda parcial do palato.

A literatura aponta que a perda de estrutura do palato provoca inúmeras consequências ao seu portador, como, por exemplo, a fala hipernasalada ${ }^{6} \mathrm{e}$ a regurgitação de alimentos da cavidade oral para a nasal $^{14}$. No presente caso clínico foi possível notar que além desta característica, a compreensão da fala também ficou comprometida, especialmente quando o paciente não usava nenhum dispositivo de tamponamento; porém, com o uso da prótese provisória e, posteriormente, da definitiva, estas características eram corrigidas.

A xerostomia é apontada com frequência como consequência do tratamento radioterápico ${ }^{14}$. Isto também pôde ser observada durante o atendimento clínico do paciente, que se queixava da sensação de "boca seca" durante as etapas clínicas e pedia que fosse deixado um copo de água próximo.

Apesar da revisão de literatura desse trabalho destacar diversas vezes que pacientes com acometimento facial apresentam maiores taxas de depressão ${ }^{7}$, o paciente desse estudo de caso teve boa aceitação com seu diagnóstico e apoio familiar, como foi possível entender durante o contato obtido com o mesmo no decorrer do tratamento. Entretanto, foi relatado que houve a perda de $23 \mathrm{~kg}$ durante o processo de tratamento e foi realizado terapia com psicólogo, mas foi dispensado após a primeira consulta, não sendo necessário o acompanhamento por parte desse, o que permite supor que o emagrecimento teve causa local (dificuldade de mastigação e deglutição) e não psicológica.

Outros profissionais foram envolvidos, como o cirurgião de cabeça e pescoço, cirurgião dentista e fonoaudiólogo, o que ratifica com a literatura que diz ser necessária uma equipe multidisciplinar para o tratamento completo do paciente ${ }^{8}$.

Segundo Carvalho ${ }^{15}$, cirurgias de tumores na região da face geralmente contraindicam cirurgias plásticas ou enxertos ósseos e teciduais, devido ao risco de uma possível recidiva que pode ser mascarada pelo tratamento, sendo a reabilitação com prótese maxilofacial uma solução viável para a maioria dos casos. Dessa forma, a indicação da prótese obturadora do caso em questão corrobora com os achados da literatura. Esta pode ser uma prótese imediata, que é realizada no ato cirúrgico para a remoção dos tecidos, ou uma prótese obturadora tardia ${ }^{16}$. Neste caso, o paciente chegou para o tratamento na universidade após a completa cicatrização da cirurgia e já utilizando uma prótese obturadora provisória, portanto, foi confeccionada uma prótese tardia.

Em pacientes que são submetidos à cirurgias como a maxilectomia, é possível notar nas áreas de enxerto de pele e, especialmente, nas não enxertadas, grande deformidade facial e diminuição na abertura bucal. Nestes casos, quando a reabilitação é realizada através de prótese reparadora tardia, não se recupera a deformidade cicatricial, não tem boa adaptação e em alguns casos é impossível de ser executada ${ }^{16}$. Embora o paciente também apresente deformidade facial na área afetada pelo tumor e pequena abertura 
de boca, o tratamento foi possível de ser realizado e reabilitou a condição oral, todavia, não foi possível devolver o contorno facial, pois o paciente continua em acompanhamento médico em função da descontinuidade da pele da face do lado esquerdo.

$\mathrm{O}$ alginato foi escolhido para as duas etapas de moldagem (anatômica e funcional) apesar de a literatura relatar que outros materiais, como a godiva $^{16}$, o silicone de adição $^{17}$ e o polissulfeto $^{18}$ podem proporcionar resultados superiores quanto à reprodução de detalhes e estabilidade dimensional. Contudo, esta indicação da literatura não foi seguida pelo fato destes materiais se tornarem extremamente rígidos após a presa, o que poderia ferir o paciente ou, ainda, ficarem retidos na área do defeito no momento da retirada do molde.

$\mathrm{O}$ número e a posição dos dentes, as condições dentárias, a altura do rebordo alveolar residual, a presença ou não do assoalho palatino influenciam na estabilidade da prótese ${ }^{16}$. A estabilidade da prótese em questão foi possível de ser obtida devido aos dentes remanescentes, os quais permitiram a retenção de uma prótese parcial removível mesmo o paciente não apresentando rebordo alveolar nem assoalho palatino do lado esquerdo, afetado pelo tumor.

Segundo Goiato et al. ${ }^{19}$, também é possível encontrar outras formas de retenção, como por implantes osseointegrados. Estes não puderam ser empregados no presente caso pelo fato de o paciente ter passado por radioterapia e por ainda estar em acompanhamento médico da área operada, o que contraindica este tipo de tratamento reabilitador.

A técnica cirúrgica para ressecção da área afetada leva sempre à necessidade de uma reconstrução, não apenas no sentido funcional, devolvendo ao paciente função mastigatória e fonética, como também no sentido de auxiliar no processo de reabilitação emocional ${ }^{16}$. Foi possível devolver a capacidade mastigatória do paciente, e também foi viabilizada a fonética durante a fala, contribuindo para a reabilitação social do mesmo.

\section{CONCLUSÃO}

Com base no caso clínico apresentado concluiu-se que a reabilitação com prótese obturadora palatina em paciente oncológico que teve parte do palato perdido por cirurgia de retirada de tumor é totalmente possível, e imprescindível ao tratamento.

As funções de fala, deglutição, mastigação e respiração do paciente ficam extremamente prejudicadas após esse tipo de cirurgia, que é muito mutiladora e traz graves sequelas na vida desse indivíduo. Restabelecer uma melhora na qualidade de vida desse paciente, que já traz uma grande carga emocional advinda não só da doença como do seu tratamento mostra-se essencial e por isso deve ser realizada, assim que possível para possibilitar sua recuperação.

Além disso, fica clara a importância de todos os profissionais envolvidos com o caso trabalharem em conjunto. É relevante mencionar o papel do cirurgião dentista como parte da equipe multidisciplinar; que deve ter conhecimento das técnicas reabilitadoras para este tipo de paciente, ainda pouco explorada na grade obrigatória dos cursos de Odontologia.

\section{REFERÊNCIAS}

1. Alonso N, Amaral DREC, Kuczynski E. Quality of life among children with cleft lips and palates: a critical review of measurement instruments. Rev Bras Cir Plast. 2011;26(4):639-44.

2. Almeida SDF, Hossotani HM, Moura DGJ. Internal carotid artery thrombosis related to trauma of palate in a child. Rev Paul Pediatr. 2012;30(1):144-47.

3. Dym RJ, Masri D, Shifteh K. Imaging of the paranasal sinuses. Oral Maxilofac Surg Clin North Am. 2012;24(2):175-89.

4. Regea CCI, Silva YPF. Carcinoma epidermóide no seio maxilar: uma revisão analítica da literatura. Sci Invest Dent. 2013;16(1):29-42.

5. Carvalho VA, Franco MHP, Kovács MJ, Liberato RP, Macieira RC, Veit MT et al. (orgs.). Temas em Psico-oncologia. São Paulo: Summus, 2008.

6. Keyf F. Obturator prosthesis for hemimaxillectomy patients. J Oral Rehabil. 2001; 28(9):821-29.

7. Irish J, Sandhu N, Simpson C, Wood R, Gilbert R, Gullane P et al. Quality of life in patients with maxillectomy prostheses. Head Neck. 2009; 31(6):813-21.

8. Kusterer LEFL, Paraguassú GM, Silva VSM, Sarmento VA. Reabilitação com obturador maxilar após cirurgia oncológica: relato de casos. Rev Cir Traumatol Buco-Maxilo-Fac. 2012;12(4):9-16

9. Sharma AB, Beumer J 3rd. Reconstruction of maxillary defects: the case for prosthetic rehabilitation. J Oral Maxillofac Surg. 2005; 63(12):1770-73.

10.Rezende JRV. Fundamentos da protese bucomaxilo-facial. 2 ed. São Paulo: Savier; 1997.

11.Goiato MC, Piovezan AP, Santos DM, Gennari Filho H, Assunção WG. Fatores que levam à utilização de uma prótese obturadora. Rev Odontol Araçatuba. 2006;27(2):101-6.

12.Silva DP, Almeida FCS, Vaccarezza GF, Brandão TB, Cazal C, Caroli A et al. Reabilitação protética de pacientes maxilectomizados, uma contribuição da odontologia e um convite à reflexão. Pesq Bras Odontoped Clin Integr. 2004;4(2):125-30.

13. Souza RP, Cordeiro FB, González FM, 
Yamashiro I, Paes Junior AJO, Tornin OS et al. Maxillary sinus carcinoma: $\mathrm{Na}$ analysis of tem cases. Radiol Bras 2006; 39(6):397-400.

14.Goiato, MC, Pesqueira AA, Ramos da Silva C, Gennari Filho H, Micheline dos Santos D. Patient satisfaction with maxillofacial prosthesis, literature review. J Plast Reconstr Aesthet Surg. 2009; 62(2): 175-80.

15.Carvalho ACGS, Catro Filho FM, Sousa FB, Magro-Filho O, Romio KB, Nogueira RLM. Immediate oral rehabilitation after partial maxillectomy: a case report. Rev cir traumatol buco-maxilo-fac. 2009; 9(2):33-8.

16. Miracca RAA, Andrade Sobrinho J, Gonçalves AJ. Reconstrução com prótese imediata pósmaxilectomia. Rev Col Bras Cir. 2007; 34(5):297-302.

17. Shibayama R, Tiossi R, Campaner M, Queiroz ME, Dallazen E. Reabilitação protética de paciente maxilectomizados: relato de caso. Rev Odont Araçatuba. 2016;37(2):9-16.

18. Bonachela WC; Kaizer OB; Kaizer ROF. Tratamento protético pós-cirúrgico de ameloblastoma de maxila. RFO UPF. 2007;12(2):68-73.

19. Goiato MC, Tabata LF, Archangelo CM, Martins Júnior M. Use of osseointegrated implants associated to retention systems in rehabilitations with oral and maxillofacial prosthesis: a review of literature. Pesq Bras Odontoped Clin Integr. 2007;7(3):331-36.

\section{CONFLITO DE INTERESSES}

Os autores declaram não haver conflitos de interesse.

\section{AUTOR PARA CORRESPONDENCIA}

\section{Marcela Filié Hadad}

marcela.haddad@unifal-mg.edu.br

Submetido em 28/08/2018

Aceito em 04/10/2018 